\title{
APLICAÇÃO DA ESPIROMETRIA DURANTE TESTE PADRÃO DE EXERCíCIO PROGRESSIVO EM ESTEIRA PARA AVALIAÇÃO DA TROCA GASOSA RESPIRATÓRIA DE EQUINOS DA RAÇA ÁRABE
}

\section{Application of spirometry during standard incremental exercise test on treadmill for respiratory gas exchange evaluation of Arabian horses}

\author{
WATANABE, M.J. ${ }^{1}$; SILVEIRA, V.F. ${ }^{2}$; MACHADO, L.P. ${ }^{2}$; YONEZAWA, L.A. ${ }^{2}$; \\ KOHOYAGAWA, A. ${ }^{2}$; THOMASSIAN, A. $^{2}$ \\ ${ }^{1}$ Cirurgia e Anestesiologia Veterinária, Cirurgia de Grandes Animais - Faculdade de Medicina Veterinária e \\ Zootecnia - UNESP - Botucatu. \\ ${ }^{2}$ Faculdade de Medicina Veterinária e Zootecnia - UNESP - Botucatu.
}

Endereço para correspondência: Marcos Jun Watanabe: watanabe@fmvz.unesp.br

\section{RESUMO}

No presente estudo avaliou-se a troca gasosa respiratória pela mensuração do consumo de oxigênio (VO2), dióxido de carbono produzido $\left(\mathrm{VCO}_{2}\right)$ e quociente respiratório $(\mathrm{R})$, e determinou-se o consumo máximo de oxigênio $\left(\mathrm{VO}_{2} \mathrm{max}\right)$ de cavalos da raça Árabe durante o teste padrão de exercício progressivo em esteira de alta velocidade. Seis equinos adultos da raça Árabe, clinicamente hígidos foram submetidos ao teste em esteira com inclinação de $6 \%$, o qual consistiu da velocidade inicial de 1,8 m/s por 5 minutos, a $4 \mathrm{~m} / \mathrm{s}$ por 3 minutos, a $6 \mathrm{~m} / \mathrm{s}$ por 2 minutos e fases a $8 \mathrm{~m} / \mathrm{s}, 9 \mathrm{~m} / \mathrm{s}, 10 \mathrm{~m} / \mathrm{s}$ e $11 \mathrm{~m} / \mathrm{s}$ por 1 minuto cada, de modo que a manta da esteira foi parada quando os cavalos não conseguiram acompanhar a velocidade da mesma. $A$ troca gasosa foi monitorada por meio da máscara de análise de gases respiratórios para equinos, nos últimos 10 segundos finais de cada mudança de velocidade e a 1, 2 e 3 minutos após o término do exercício. Durante o exercício há elevação do $\mathrm{VO}_{2}$ e $\mathrm{VCO}_{2}$ com relação linear conforme a intensidade de exercício. $\mathrm{O} \mathrm{VO}_{2} \mathrm{max}$ dos cavalos da raça Árabe é de $114,9 \mathrm{~mL} / \mathrm{kg} / \mathrm{min}$. O quociente respiratório eleva-se para valores maiores que 1,0 a partir da velocidade de $9,0 \mathrm{~m} / \mathrm{s}$, indicando o predomínio do metabolismo anaeróbico, e mantêm-se ainda elevado no período pós-exercício.

Palavras-chave: consumo de oxigênio, equino, metabolismo, quociente respiratório, sistema respiratório

\begin{abstract}
The current study evaluated equine gas exchange responses through spirometry, by measuring oxygen uptake $\left(\mathrm{VO}_{2}\right)$, carbon dioxide production $\left(\mathrm{VCO}_{2}\right)$, respiratory exchange ratio $(\mathrm{R})$ and maximum oxygen uptake $\left(\mathrm{VO}_{2} \mathrm{max}\right)$ of Arabian horses during a standard incremental exercise test performed on a high-speed treadmill. Six clinically healthy Arabian horses were submitted to a standard incremental exercise test, performed on a high-speed treadmill at a $6 \%$ slope, and initial speed of $1,8 \mathrm{~m} . \mathrm{s}-1$ for 5 minutes, then 4,0 m.s-1 for 3 minutes, 6,0 m.s-1 for 2 minutes and 8,0 m.s-1, 9,0 m.s-1, 10,0 m.s-1 and 11,0 m.s-1 for one minute for each of these speed. The end of the exercise test was defined as the point in which the horse was no longer able to keep pace with the treadmill. Gas exchange was measured through respiratory analyses horse mask on the last 10 seconds at the end of each speed transition and at 1, 2 and 3 minutes after the end of the exercise, defined as experiment moments. During exercise, it was noticed an increase on both $\mathrm{VO}_{2}$ and $\mathrm{VCO}_{2}$ with linear relationship between exercise and speed. Arabian horses $\mathrm{VO}_{2}$ max was 114,9 $\mathrm{mL} . \mathrm{kg}-1 . \mathrm{min}-1$. The respiratory exchange ratio increased over 1,0 by the speed of $9,0 \mathrm{~m} . \mathrm{s}-1$, indicating the preponderance of the anaerobic metabolism, and remains at a high level on the post-exercise period.
\end{abstract}

Key words: aerobic capacity, equine, oxygen uptake, respiratory system. 


\section{INTRODUÇÃO}

Pesquisas realizadas com cavalos atletas indicam que o sistema respiratório pode ser o fator limitante ao máximo desempenho mesmo em cavalos hígidos, sendo que o conhecimento dos detalhes de sua função respiratória é essencial para sua adequada avaliação.

Os principais objetivos dos testes físicos de exercícios são estudar a fisiologia do exercício, avaliar parâmetros metabólicos relativos ao diagnóstico de perda de rendimento atlético, determinação do potencial de desempenho e monitoramento do treinamento. Assim, os parâmetros obtidos durante os testes físicos podem fornecer informações a respeito tanto do metabolismo aeróbico quanto do anaeróbico, bem como das respostas fisiológicas frente ao exercício (Seeherman e Morris, 1990a).

A espirometria realizada durante um teste de exercício físico pode ser considerada como uma forma de avaliar a função cardiorrespiratória, pela qual são mensurados volumes de ar e concentrações de oxigênio $\left(\mathrm{O}_{2}\right)$ e dióxido de carbono $\left(\mathrm{CO}_{2}\right)$ inspirados e expirados em função do tempo (Miller et al., 2005). Por exemplo, o consumo de oxigênio $\left(\mathrm{VO}_{2}\right)$ de um cavalo refere-se à taxa na qual o oxigênio é utilizado pela mitocôndria no músculo (Holcombe, 2006) e em cavalos em repouso está em torno de 2 a 3 $\mathrm{mL} / \mathrm{min} / \mathrm{kg}$ (Evans, 2000). O quociente respiratório $(R)$ é determinado pela mensuração das concentrações de $\mathrm{CO}_{2}$ e $\mathrm{O}_{2}$ nos gases expirados, de maneira que 0 $\mathrm{R}$ corresponde a relação das concentrações de $\mathrm{CO}_{2}$ produzido/ $/ \mathrm{O}_{2}$ utilizado.

O volume máximo de oxigênio utilizado pelo cavalo durante um exercício máximo é definido como consumo máximo de oxigênio $\left(\mathrm{VO}_{2} \max \right)$ e pode ser obtido por meio do monitoramento constante do $\mathrm{VO}_{2}$ durante 0 teste padrão de exercício progressivo (Derman e Noakes, 1994). Em atletas humanos, o $\mathrm{VO}_{2}$ max é considerado um índice confiável pelo qual se avalia a capacidade de indivíduos para realizar exercícios aeróbicos. Em cavalos da raça $\mathrm{PSI}, \mathrm{O} \mathrm{VO}_{2} \mathrm{max}$ foi relacionado a menores intervalos de tempos para percorrer a distância de 1000 a 2000 metros (Hodgson e Rose, 1994) e Gauvreau et al. (1995), observaram que os cavalos com altos VO2max apresentaram melhor desempenho atlético na modalidade de corrida de trote.

Rose et al. (1995), compararam índices de desempenho atlético entre cavalos da raça $\mathrm{PSI}$ e da raça de corrida de trote, e observaram maior capacidade aeróbica nos cavalos $\mathrm{PSI}$, relacionada com seu maior $\mathrm{VO}_{2}$ max. Prince et al. (2002) compararam as respostas metabólicas de cavalos da raça PSI e Árabes durante diferentes testes de exercício, e observaram nos animais da raça $\mathrm{PSI}$, valores maiores de $\mathrm{VO}_{2} \mathrm{max}$, da velocidade para o $\mathrm{VO}_{2}$ max e do tempo total de exercício durante o teste de exercício progressivo.

Considerando que métodos de avaliação do sistema respiratório podem contribuir, tanto para o entendimento da complexa integração deste sistema com a atividade física quanto para a detecção de alterações que podem influenciar 0 desempenho atlético competitivo, esta pesquisa teve como objetivo avaliar parâmetros de troca gasosa respiratória $\left(\mathrm{VO}_{2}, \mathrm{VCO}_{2}\right.$ e $\left.\mathrm{R}\right)$ e determinar o $\mathrm{VO}_{2} \max$, de cavalos da raça Árabe durante o teste padrão de exercício progressivo em esteira de alta velocidade.

\section{MATERIAL E MÉTODOS}

Seis equinos da raça Árabe, dois machos castrados e quatro fêmeas, idade entre seis e 12 anos, $350 \pm 24,1 \mathrm{~kg}$ de peso e sem atividade física controlada por pelo menos três meses, foram submetidos aos exames: físico, laboratoriais, de claudicação, eletrocardiográfico, ecocardiográfico, vídeo-endoscópico do trato respiratório 
anterior e laringoscópico com o equino durante o exercício sobre a manta da esteira. Por meio destes, verificaram-se possíveis alterações que pudessem interferir nas respostas fisiológicas frente ao exercício.

Após a verificação da higidez, os animais foram submetidos ao período de adaptação à locomoção sobre a manta da esteira de alta velocidade (Mustang 2200 KAGRA, Suíça), a presença de uma máscara espirométrica para equinos $e$, ao ambiente e procedimentos de realização dos testes de exercício por um período de 10 dias. Durante o experimento os animais foram mantidos em piquetes com água ad libitum e alimentados com feno de capim coast-cross, suplementados com ração comercial para equinos (Triumph - SOCIL, Brasil) e composto mineral (Mineral ADE ROYAL HORSE, Brasil).

Previamente aos testes físicos de exercício, o sistema de análise de gases respiratórios (MetaVet - CORTEX, Alemanha) foi calibrado utilizando-se mistura de gases com concentrações conhecidas $\left(15 \% \mathrm{O}_{2}, 5 \% \mathrm{CO}_{2}\right.$ balanceado com $\mathrm{N}_{2}$ ) e programa específico, conforme orientação do fabricante (MetaSoft CORTEX, Alemanha). Os testes padrões de exercício progressivo foram realizados entre 6:00 e 7:00h da manhã, submetendo um cavalo por dia. O exercício consistiu de inclinação da esteira a $6 \%$, velocidade a $1,8 \mathrm{~m} / \mathrm{s}$ por cinco minutos, a $4,0 \mathrm{~m} / \mathrm{s}$ por três minutos, a $6,0 \mathrm{~m} / \mathrm{s}$ por dois minutos e fases a 8,$0 ; 9,0 ; 10,0$ e $11,0 \mathrm{~m} / \mathrm{s}$ por um minuto cada, de modo que a manta da esteira foi parada quando os cavalos não conseguiram acompanhar a sua velocidade mesmo sendo estimulados (Watanabe et al., 2006). No intervalo dos dez segundos finais de cada fase de mudança de velocidade e aos 1,2 e 3 min após o término do exercício, foram aferidos $\mathrm{O} \mathrm{VO}_{2}$, o $\mathrm{VCO}_{2}$ e o R por meio de um tubo coletor de amostras de gases respiratórios acoplado à máscara espirométrica específica para equinos, onde as concentrações de $\mathrm{O}_{2}$ e $\mathrm{CO}_{2}$ do ar foram determinadas por uma central processadora de dados espirométricos que utiliza sensor de óxido de zircônio para a determinação do $\mathrm{O}_{2}$ e sensor infravermelho para $\mathrm{O} \mathrm{CO}_{2}$. As mensurações durante $\mathrm{o}$ exercício foram realizadas sem a parada da manta da esteira e durante todo o teste os cavalos receberam fluxo de ar de 2,2 m/s gerado por ventilador posicionado frontalmente à esteira.

Os resultados foram analisados mediante aplicação do teste não paramétrico de Friedman. Quando detectadas diferenças significativas das variáveis nos momentos estudados, aplicou-se o teste não paramétrico de comparações múltiplas (Zar, 1999), visando avaliar em quais momentos os resultados diferiram. Considerou-se, para todos os testes, um nível de $5 \%$ de significância e o programa utilizado para execução da análise estatística foi o $R$ ( $R$ Development Core Team, 2007).

\section{RESULTADOS E DISCUSSÃO}

Quatro dos seis cavalos completaram a fase de velocidade de exercício de 11,0 $\mathrm{m} / \mathrm{s}$, não sendo esta submetida a análise estatística adotada. Os valores das variáveis de troca gasosa estão expostos na Tabela 1.

Verificou-se elevação significativa dos valores do consumo de oxigênio apenas para a velocidade de $10 \mathrm{~m} / \mathrm{s}$. Porém, avaliando o comportamento dos valores do $\mathrm{VO}_{2}$ (Figura 1) verificou-se tendência a elevação linear até as velocidades de exercício submáximo, corroborando ao citado por Hörnicke et al. (1983), Seeherman e Morris (1990b), Rose et al. (1990), Eaton (1994) e Evans (2000). O comportamento do $\mathrm{VO}_{2}$ durante o exercício foi decorrente tanto da elevação da distribuição de $\mathrm{O}_{2}$ para os tecidos, representado pelo débito cardíaco e concentração de hemoglobina, quanto do consumo de $\mathrm{O}_{2}$ pela musculatura 
Tabela 1 - Médias, desvios-padrão e análise estatística do consumo de oxigênio $\left(\mathrm{VO}_{2}\right.$ em $\left.\mathrm{L} / \mathrm{min}\right)$, do $\mathrm{CO}_{2}$ produzido $\left(\mathrm{VCO}_{2} \mathrm{em} \mathrm{L} / \mathrm{min}\right)$ e do quociente respiratório $\left(\mathrm{R}=\mathrm{VCO}_{2} / \mathrm{VO}_{2}\right)$, dos equinos da raça árabe durante 0 teste padrão de exercício progressivo

\begin{tabular}{|c|c|c|c|c|c|c|c|c|c|c|}
\hline & \multicolumn{7}{|c|}{ Velocidade de exercício (m/s) } & \multicolumn{3}{|c|}{ Pós-exercício (min) } \\
\hline & 1,8 & 4,0 & 6,0 & 8,0 & 9,0 & 10,0 & 11,0 & 1 & 2 & 3 \\
\hline $\begin{array}{c}\mathrm{VO}_{2} \\
\text { (L/min) }\end{array}$ & $\begin{array}{l}10,4^{\mathrm{cb}} \\
\pm 1,9\end{array}$ & $\begin{array}{c}20,6^{\text {cba }} \\
\pm 1,8\end{array}$ & $\begin{array}{c}26,3^{\text {cba }} \\
\pm 5,2\end{array}$ & $\begin{array}{c}30,6^{\text {ba }} \\
\pm 3,7\end{array}$ & $\begin{array}{c}32,7^{\text {ba }} \\
\pm 4,5\end{array}$ & $\begin{array}{c}37,6^{a} \\
\pm 6,6\end{array}$ & $\begin{array}{l}43,7^{*} \\
\pm 6,0\end{array}$ & $\begin{array}{l}9,4^{\mathrm{cb}} \\
\pm 1,7\end{array}$ & $\begin{array}{l}5,0^{c} \\
\pm 0,4\end{array}$ & $\begin{array}{l}4,9^{c} \\
\pm 1,7\end{array}$ \\
\hline $\begin{array}{c}\mathrm{VCO}_{2} \\
(\mathrm{~L} / \mathrm{min})\end{array}$ & $\begin{array}{l}9,0^{\mathrm{cb}} \\
\pm 1,5\end{array}$ & $\begin{array}{c}18,3^{\mathrm{cba}} \\
\pm 2,0\end{array}$ & $\begin{array}{c}24,9^{b a} \\
\pm 3,0\end{array}$ & $\begin{array}{c}29,5^{\mathrm{a}} \\
\pm 1,8\end{array}$ & $\begin{array}{c}35,3^{a} \\
\pm 4,1\end{array}$ & $\begin{array}{c}42,2^{a} \\
\pm 6,0\end{array}$ & $\begin{array}{l}49,0^{*} \\
\pm 3,4\end{array}$ & $\begin{array}{c}18,7^{\mathrm{cba}} \\
\pm 3,1\end{array}$ & $\begin{array}{l}9,1^{c b} \\
\pm 1,0\end{array}$ & $\begin{array}{l}7,2^{c} \\
\pm 0,9\end{array}$ \\
\hline $\mathrm{R}$ & $\begin{array}{l}0,89^{b} \\
\pm 0,06\end{array}$ & $\begin{array}{l}0,92^{b} \\
\pm 0,04\end{array}$ & $\begin{array}{l}0,99 \\
\pm 0,08\end{array}$ & $\begin{array}{l}0,99 \text { ba } \\
\pm 0,07\end{array}$ & $\begin{array}{l}1,12^{\text {ba }} \\
\pm 0,09\end{array}$ & $\begin{array}{l}1,18^{b a} \\
\pm 0,09\end{array}$ & $\begin{array}{c}1,22^{*} \\
\pm 0,07\end{array}$ & $\begin{array}{l}2,07^{a} \\
\pm 0,23\end{array}$ & $\begin{array}{r}1,89^{a} \\
\pm 0,34\end{array}$ & $\begin{array}{r}1,58^{a} \\
\pm 0,36\end{array}$ \\
\hline
\end{tabular}

Momentos com letras iguais indicam que os valores em tais momentos não diferem entre si de forma significativa $(P>0,05)$.

* Valor não avaliado estatisticamente pelo fato de apenas quatro dos seis cavalos completarem esta fase.

esquelética impostas pelas cargas de trabalho crescentes (Holcombe, 2006).

Por meio da avaliação dos valores do $\mathrm{VO}_{2}$ durante as fases de velocidade máxima observou-se ainda evidências de uma relação linear com a velocidade, similarmente ao observado por Butler et al. (1993). Entretanto, estes resultados diferiram da maioria das pesquisas que citam a formação de um platô em altas intensidades de exercício, que seria definido como $\mathrm{VO}_{2}$ max (Rose et al., 1990; Eaton, 1994; Evans, 2000 e McDonough et al., 2002a). Seeherman e Morris (1990b) relacionaram a não formação do platô com a influência de fatores periféricos, como o comprometimento da função muscular

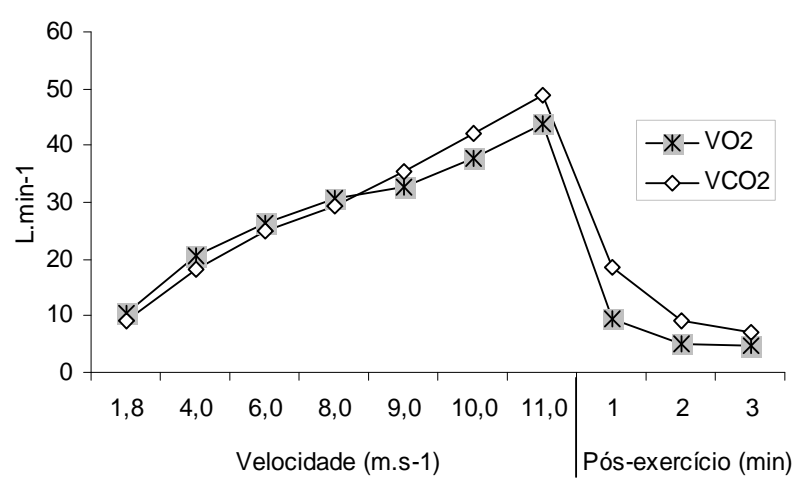

Figura 1 - Representação gráfica das médias do consumo de oxigênio $\left(\mathrm{VO}_{2} \mathrm{em} \mathrm{L} / \mathrm{min}\right)$ e do dióxido de carbono produzido $\left(\mathrm{VCO}_{2} \mathrm{em} \mathrm{L} / \mathrm{min}\right)$ dos equinos da raça árabe durante o teste padrão de exercício progressivo. antes que o cavalo alcançasse o $\mathrm{VO}_{2} \max$.

Vários estudos utilizam os testes de exercício progressivo em esteira para a determinação do $\mathrm{VO}_{2} \max$, mas com exercícios sendo realizados com inclinação de em torno de $10 \%$, correspondente a $5,71^{\circ}$. No presente estudo, utilizou-se inclinação de $6 \%$, instigando, com isso, a possibilidade da menor inclinação influenciar a obtenção do platô do $\mathrm{VO}_{2}$ em altas velocidades. Porém, McDonough et al. (2002a) submeteram seis cavalos PSI ao teste de exercício progressivo com inclinação de $10 \%$ e sem inclinação, e verificaram o mesmo comportamento da resposta do $\mathrm{VO}_{2}$ conforme a velocidade de exercício, com formação do platô nas duas situações, porém uma elevação de cerca de $20 \%$ do $\mathrm{VO}_{2} \max$ foi notada quando 0 exercício foi realizado com inclinação.

Assim, a diferença da curva do $\mathrm{VO}_{2}$ nos testes padrões de exercício progressivo seria justificada pelo fato de haver uma porcentagem de animais que não demonstram o platô do $\mathrm{VO}_{2}$ durante o teste de exercício progressivo, como observado por Seeherman e Morris (1990b) que verificaram a formação de platô em seis de 10 cavalos.

Como o grupo experimental foi composto por seis animais, a divisão entre cavalos que tenderam a formação do platô ficou comprometida, mas analisando os cavalos 1, 2 e 5, a diferença do $\mathrm{VO}_{2}$ entre 
Tabela 2 - Valores individuais do peso $(\mathrm{kg})$, do consumo de oxigênio $\left(\mathrm{VO}_{2} \mathrm{em} \mathrm{mL} / \mathrm{kg} / \mathrm{min}\right)$ e do consumo máximo de oxigênio $\left(\mathrm{VO}_{2 \max } \mathrm{em} \mathrm{mL} / \mathrm{kg} / \mathrm{min}\right)$ dos equinos da raça árabe durante o teste padrão de exercício progressivo

\begin{tabular}{ccccccccc}
\hline & Peso & \multicolumn{7}{c}{ Velocidade de exercício (m/s) } \\
\cline { 3 - 8 } & $(\mathrm{kg})$ & 1,8 & 4,0 & 6,0 & 8,0 & 9,0 & 10,0 & 11,0 \\
\hline 1 & 358 & 21,8 & 57,9 & 70,3 & 80,2 & 81,6 & $88,0^{*}$ & \\
2 & 329 & 35,3 & 61,4 & 54,0 & 89,7 & 105,7 & 103,3 & $109,4^{*}$ \\
3 & 350 & 28,2 & 58,6 & 90,3 & 91,8 & 99,9 & 120,6 & $133,4^{*}$ \\
4 & 316 & 28,7 & 55,0 & 91,2 & 91,8 & 91,4 & 120,4 & $133,9^{*}$ \\
5 & 365 & 35,7 & 62,7 & 84,2 & 102,3 & 108,9 & 132,2 & $136,3^{*}$ \\
6 & 382 & 43,6 & 59,7 & 63,5 & 69,1 & 69,2 & $88,2^{*}$ &
\end{tabular}

* Valores individuais do consumo máximo de oxigênio em $\mathrm{mL} / \mathrm{kg} / \mathrm{min}$.

a velocidade máxima e a anterior foi de 6,$4 ; 6,1$ e $4,1 \mathrm{~mL} / \mathrm{kg} / \mathrm{min}$, respectivamente (Tabela 2), sugerindo a formação do platô nestes animais em virtude da pequena elevação do $\mathrm{VO}_{2}$ entre as velocidades.

A determinação do $\mathrm{VO}_{2} \max$ seguiu o critério adotado para a determinação do $\mathrm{VO}_{2}$ max com base na observação do maior valor de $\mathrm{VO}_{2}$ observado durante o exercício (pico de $\mathrm{VO}_{2}$ ) com valores crescentes do $\mathrm{VCO}_{2}$ e do quociente respiratório maiores que 1,2 (Seeherman e Morris, 1990a). Assim, a média do $\mathrm{VO}_{2}$ max de 114,9 22,9 $\mathrm{mL} / \mathrm{kg} / \mathrm{min}$ foi calculada a partir do valor máximo de cada cavalo durante o exercício (tabela 2), valor este similar ao observado por Tyler et al. (1996a) que relataram valor de $117 \pm 2 \mathrm{~mL} / \mathrm{kg} / \mathrm{min}$. Valores mais elevados foram obtidos por Tyler et al. (1996b) que determinaram $\mathrm{VO}_{2} \mathrm{max}$ de $135,1 \pm 2 \mathrm{~mL} / \mathrm{kg} / \mathrm{min}$. Seeherman e Morris (1990b) observaram $\mathrm{VO}_{2} \max$ ainda mais elevados em 10 cavalos da raça PSI, sendo este de $161,9 \pm 12,9 \mathrm{~mL} / \mathrm{kg} / \mathrm{min}$.

$\mathrm{O}$ menor valor do $\mathrm{VO}_{2}$ max observado na presente pesquisa pode estar relacionado tanto com a diferença, de $6 \%$ para $10 \%$ de inclinação da esteira adotada nas pesquisas (McDonough et al., 2002a), quanto com o grupo de cavalos utilizados principalmente com relação à idade, peso, raça e estado de condicionamento físico.

As características do grupo experimental podem ter influenciado o $\mathrm{VO}_{2} \max$ neste estudo. Os cavalos apresentavam idade variando entre seis e 12 anos, enquanto grande parte das pesquisas utilizou animais jovens que obtiveram $\mathrm{VO}_{2} \max$ mais elevados. Beltros et al. (2002) constataram que animais velhos possuiam menores $\mathrm{VO}_{2}$ max (média de $83,2 \mathrm{~mL} / \mathrm{kg} / \mathrm{min}$ ) em comparação aos jovens (média de $116 \mathrm{~mL} / \mathrm{kg} / \mathrm{min}$ ).

Acredita-se também que o tamanho do animal (massa corporal) possa influenciar o $\mathrm{VO}_{2}$ max. Katz et al. (2005) relataram baixos valores de $\mathrm{VO}_{2}$ max em pôneis $(92,0$ $\pm 3,8 \mathrm{~mL} / \mathrm{kg} / \mathrm{min}$ ) associando esses valores à baixa capacidade aeróbica desses animais frente à massa específica.

Outro fator a ser considerado é a predominância da utilização de cavalos da raça PSI nas pesquisas, sendo que estes possuem características inerentes à raça $e$ ao tipo de modalidade esportiva desempenhada. Em relação à influência da raça no $\mathrm{VO}_{2} \max$, Prince et al. (2002) concluíram que cavalos PSI possuem maior capacidade aeróbica quando 
comparados aos cavalos da raça Árabe, contribuindo desta forma para um melhor potencial de desempenho em exercícios de alta intensidade.

Quando avaliado o $\mathrm{VCO}_{2}$, similarmente ao $\mathrm{VO}_{2}$, apresentou comportamento de elevação linear conforme a intensidade de exercício corroborando com a pesquisa de Seeherman e Morris (1990b) e de McDonough et al. (2002b). Esta elevação foi reflexo da remoção do $\mathrm{CO}_{2}$ tecidual produzido pelo metabolismo energético (Mainwood e Renaud, 1985).

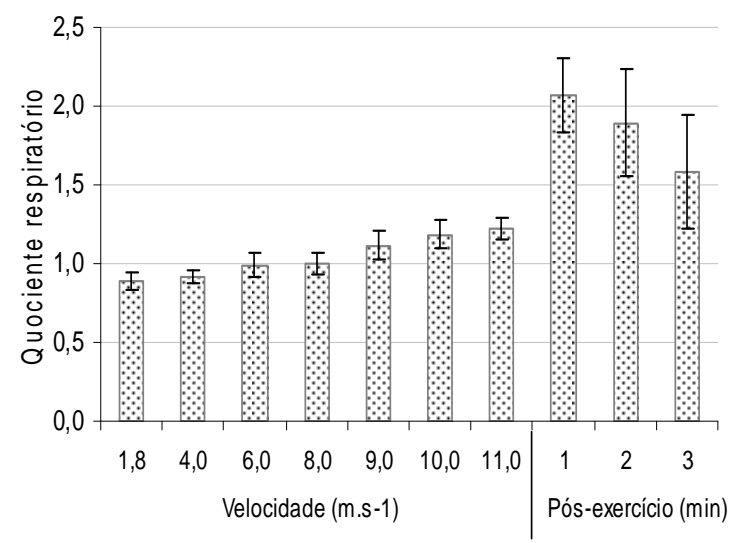

Figura 2 - Médias e desvios-padrão do quociente respiratório $(R)$, dos equinos da raça árabe durante o teste padrão de exercício progressivo.

Durante o exercício, não foi verificada alteração significativa do quociente respiratório (R). Notaram-se elevações significativas apenas nos momentos correspondentes a 2 e $3 \mathrm{~min}$ após 0 término do exercício. No entanto, verificouse tendência ao aumento dos valores do $R$ ao longo do exercício (Figura 2). Porém, a partir da velocidade de $9 \mathrm{~m} / \mathrm{s}$ a quantidade de $\mathrm{CO}_{2}$ produzido superou a de $\mathrm{O}_{2}$ utilizado, refletindo desta maneira na mudança do tipo de metabolismo ao longo do exercício. Provavelmente, até a velocidade de $8 \mathrm{~m} / \mathrm{s}$, o exercício foi mantido predominantemente pela oxidação de carboidratos e gordura (Marlin e Nankervis, 2002). Já na velocidade $9 \mathrm{~m} / \mathrm{s}$, com valor médio de $R$ de $1,12 \pm 0,09$, o metabolismo predominante foi 0 anaeróbico com produção de lactato. O valor máximo observado durante o exercício referiu-se a velocidade de $11 \mathrm{~m} / \mathrm{s}$ com valor de 1,22 \pm 0,07 , valor semelhante ao descrito por Seeherman e Morris (1990b) que verificaram $R$ de 1,2 ao final do teste de exercício progressivo.

Mesmo com o declínio dos valores de $\mathrm{VO}_{2}$ e $\mathrm{VCO}_{2}$ após o término do exercício, observaram-se maiores valores de $\mathrm{R}$ neste período, como resultado de uma diminuição mais acentuada do $\mathrm{VO}_{2}$ relativamente ao $\mathrm{VCO}_{2}$. Comportamento similar do $\mathrm{R}$ pósexercício também foi observado na pesquisa de Seeherman e Morris (1990a).

\section{CONCLUSÕES}

Durante o exercício há elevação do $\mathrm{VO}_{2}$ e $\mathrm{VCO}_{2}$, com relação linear conforme a intensidade de exercício. $\mathrm{O} \mathrm{VO}_{2} \max$ dos cavalos da raça Árabe foi de 114,9 $\mathrm{mL} / \mathrm{kg} / \mathrm{min}$. O quociente respiratório elevase para valores maiores que 1,0 a partir da velocidade de $9,0 \mathrm{~m} / \mathrm{s}$, indicando 0 predomínio do metabolismo anaeróbico, e mantêm-se ainda elevado no período pósexercício.

\section{AGRADECIMENTOS}

À Fundação de Amparo à Pesquisa do Estado de São Paulo - FAPESP, pelo Auxílio à Pesquisa concedido, processo FAPESP no 04/01715-4.

\section{COMITÊ DE ÉTICA E BEM ESTAR ANIMAL}

O presente estudo foi aprovado de acordo com os princípios Éticos na Experimentação Animal (COBEA), protocolo número 45/2004 - CEEA.

\section{REFERÊNCIAS}

BELTROS, C.L. et al. Effects of ageing and training on maximal heart rate and $\mathrm{VO}_{2 \max }$.

Equine Veterinary Journal - Equine 
Exercise Physiology 6, v.34, suppl., p.100-105, 2002.

BUTLER, P.J. et al. Respiratory and cardiovascular adjustments during exercise of increasing intensity and during recovery in Thoroughbred racehorses. The Journal of Experimental Biology, Cambridge, v.179, n.1, p.159-180, 1993.

DERMAN, K.D.; NOAKES, T.D. Comparative aspects of exercise physiology. In: HODGSON, D. R.; ROSE, R. J. The Athletic Horse. Philadelphia: W. B. Saunders, 1994. p.15-25.

EATON, M.D. Energetics and Performance. In: HODGSON, D. R.; ROSE, R. J. The Athletic Horse. Philadelphia: W. B. Saunders, 1994. p.49-61.

EVANS, D.L. Training and Fitness in Athletic Horses. Kingston: Rural Industries Research and Development Corporation, 2000. 87p.

GAUVREAU, G.M.; STAEMFLI, H.; MCCUTCHEON, L.J. et al. Comparison of aerobic capacity between racing standardbred horses. Journal of Applied Physiology, v.78, n.4, p. 1447-1451, 1995. HODGSON, D. R.; ROSE, R. J. Evaluation of Performance Potential. In: The

Athletic Horse. Philadelphia: W. B. Saunders, 1994. p.231-243.

HOLCOMBE, S.J. Pulmonary function in Racehorses. In: Annual Convention of the American Association of Equine Practitioners, 8., 2006, Rome.

Proceedings... Rome, 2006. p.19-21.

HÖRNICKE, H. et al. Respiration in exercising horses. In: International Conference on Equine Exercise Physiology - Equine Exercise Physiology 2, 1983, Cambridge. Proceedings ... 1983. p.7-16.

KATZ, L.M. et al. Ventilatory responses of ponies and horses to exercise. Equine and Comparative Exercise Physiology, Cambridge, v.2, n.4, p. 229-240, 2005.
MAINWOOD, G. W.; RENAUD, J. M. The effect of acid-base balance on fatigue of skeletal muscle. Canadian Journal Physiology Pharmacology. v.63, n.403, 1985.

MARLIN, D.J.; NANKERVIS, K. Exercise testing. In: Equine Exercise

Physiology, Blackwell Publishing, 2002. p.211-244.

McDONOUGH, P.; KINDIG, C.A.; ERICKSON, H.H. et al. Mechanistic basis for the gas exchange threshold in Thoroughbred horses. Journal of Applied Physiology, v.92, n.4, p.1499-1505, 2002a.

McDONOUGH, P.; KINDIG, C.A.; RAMSEL, C. et al. The effect of treadmill incline on maximal oxygen uptake, gas exchange and metabolic response to exercise in the horse. Experimental Physiology, v. 84, n. 4, p.499-506, $2002 b$.

MILLER, M.R.; HANKINSON, J.; BRUSASCO, V. et al. Standardization of Spirometry. European Respiratory Journal, v. 26, n.2, p.319-338, 2005.

PRINCE, A.; GEOR, R.; HARRIS, P. et al. Comparison of the metabolic responses of trained Arabians and Thoroughbreds during high-and low-intensity exercise. Equine Veterinary Journal - Equine Exercise Physiology 6, v.34, suppl., p.95-99, 2002.

R DEVELOPMENT CORE TEAM. R: A language and environment for statistical computing. R Foundation for Statistical Computing, 2007. Disponível em: $<$ http://www.R-project.org>.

ROSE, R.J.; HODGSON, D.R.; BAYLY, W.M. et al. Kinetics of $\mathrm{VO}_{2}$ e $\mathrm{VCO}_{2}$ in the horse and comparison of five methods for determination of maximum oxygen uptake. Equine Veterinary Journal, v.9, suppl., p.39-46, 1990.

ROSE, R.J.; KING, C.M.; EVANS, D.L. et al. Indices of exercise capacity in horses presented for poor performance. Equine 
Veterinary Journal - Equine Exercise Physiology 4, suppl., v.18, p.418-421, 1995.

SEEHERMAN, H.J.; MORRIS, E.A. Methodology and repeatability of the standardized treadmill exercise test for clinical evaluation of fitness in horse.

Equine Veterinary Journal, v.9, suppl., p. 20-25, 1990a.

SEEHERMAN, H. J.; MORRIS, E. A. Application of a standardized treadmill exercise test for clinical evaluation of fitness in 10 thoroughbred racehorses. Equine Veterinary Journal, v.9, suppl., p. 26-34, 1990b.

TYLER, C.M.; GOLLAND, L.C.; EVANS, D.L. et al. Changes in maximum oxygen uptake during prolonged training, overtraining, and detraining in horses. Journal of Applied Physiology, v.81, n.5, p.2244-2249, 1996a.
TYLER, C.M.; HODGSON, D.R.; ROSE, R.J. Effect of a warm-up on energy supply during high intensity exercise in horses. Equine Veterinary Journal., v.28, n.2, p.117-120, 1996b.

WATANABE, M.J.; THOMASSIAN, A.; TEIXEIRA-NETO, F.J. et al. Alterações do $\mathrm{pH}$, da $\mathrm{PO}_{2}$ e da $\mathrm{PCO}_{2}$ arteriais e da concentração de lactato sanguíneo de cavalos da raça Árabe durante exercício em esteira de alta velocidade. Arquivo Brasileiro de Medicina Veterinária e Zootecnia, v. 58, n. 3, p. 320-326, 2006.

ZAR, J.H. Biostatistical analysis. 4.ed. New Jersey : Prentice Hall, 1999. 663p. 